\title{
A Case Report of Vitiligo Induced by Alexandrite Hair Removal Laser
}

\author{
Azzam Alkhalifah \\ Dermatology Unit, Department of Medicine, Unaizah College of Medicine and Medical \\ Sciences, Qassim University, Qassim, Saudi Arabia
}

\section{Keywords}

Vitiligo $\cdot$ Koebner phenomenon · Alexandrite laser $\cdot$ Laser hair removal

\begin{abstract}
Vitiligo is one of the disorders where we usually see Koebner lesions. Lasers target a chromophore in the skin to deliver their energy. Their effect is theoretically considered as injury and may induce Koebner lesions in vitiligo patients. Few cases were reported with the different kinds of laser, including laser hair removal (LHR). No cases have ever been reported of vitiligo lesions induced by Alexandrite LHR. Here we present a case report of a young female patient who had never had vitiligo until she received a 755-nm LHR treatment. These vitiligo lesions were completely treated with NB-UVB with no recurrence until 4 years later when she received another 755-nm LHR treatment session with a different machine, which lead to new vitiligo patches only on the areas that received the laser.
\end{abstract}

\section{Introduction}

Vitiligo, psoriasis, and lichen planus usually manifest Koebner lesions following skin injuries. Laser hair removal (LHR) and other laser devices work by targeting a chromophore in the skin. For hair removal, the chromophore is the melanin of hair shafts, while the actual target is the hair follicle and its bulge. Its destruction is achieved through the heat produced by the hair shaft when it absorbs laser energy [1]. Both the rupture of the chromophore and the heat that reaches the surrounding tissues are sorts of insults to the skin. In vitiligo patients, we are usually afraid that this kind of skin aggression may lead to Koebner lesions. Alexandrite LHR, the most commonly used LHR, has never been reported to cause vitiligo. In contrary, it was reported to be safe in 2 case reports $[2,3]$. 


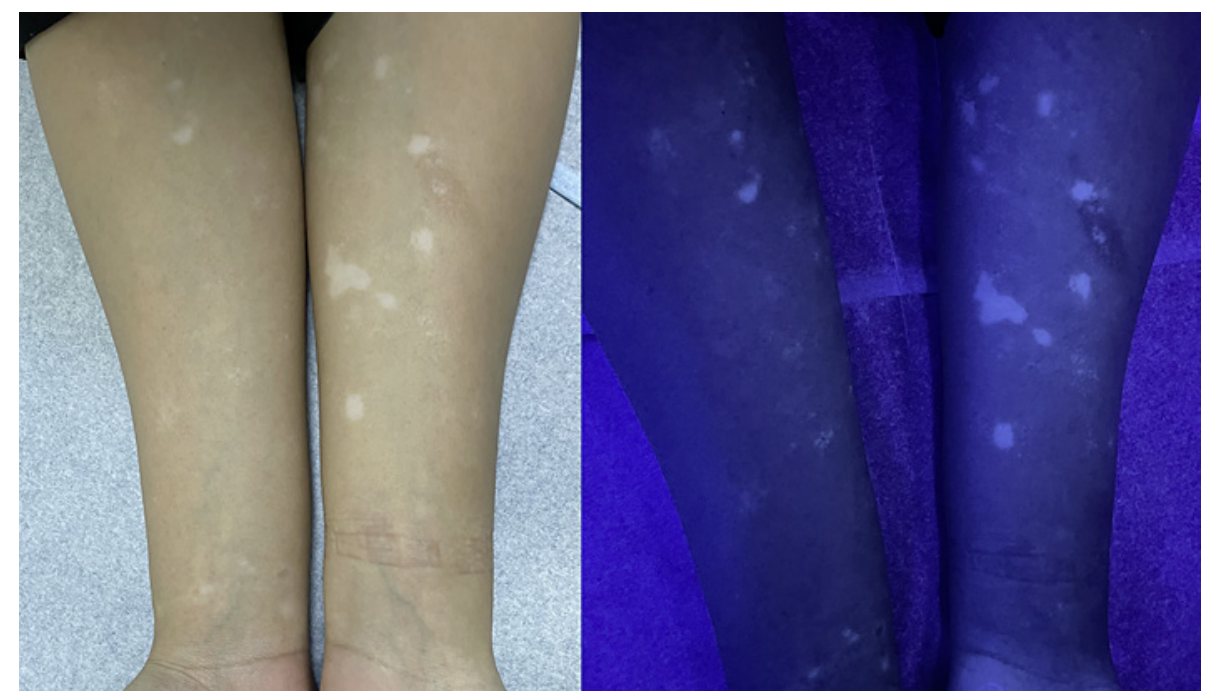

Fig. 1. Laser-induced vitiligo patches on both forearms (clinical and wood's lamp) persisting despite 3-month treatment with NB-UVB and tacrolimus $0.1 \%$ ointment.

\section{Case Report/Case Presentation}

A 28-year-old healthy woman came recently to our clinic complaining of whitish patches on her forearms and legs, 4 weeks after a LHR session (Deka Synchro Replay, $755 \mathrm{~nm}$, parameters unknown). She had the same complaint 4 years ago when she had a full body LHR session (GentleLase Pro $755 \mathrm{~nm}$, parameters unknown). At that time, the patches appeared all over her body, and improved only after 9 months of NB-UVB. This year, she has done a LHR session only on her legs and forearms, and the patches appeared only on those areas. Under wood's lamp, the patches appeared completely depigmented. There was no patches on other areas. She denied having any burns or skin peeling after the laser. We started treating her with topical tacrolimus $0.1 \%$ with biweekly NB-UVB. Three months later, she still has depigmented patches (shown in Fig. 1) with some peripheral repigmentation (shown in Fig. 2). She is still on the same treatment protocol.

\section{Discussion/Conclusion}

The patient, and her family, had never been diagnosed with vitiligo before having these 2 episodes. The laser-induced vitiligo lesions appeared gradually over 4 weeks, without any signs of postlaser inflammation, burn, or peeling. The recurrence and the long persistence despite phototherapy are not in favor of a simple postinflammatory hypopigmentation. In addition, after 3 months of NB-UVB, repigmentation started at the periphery, without central pigmentation. This confirms that the patches are devoid of melanocytes. As the patient refused the biopsy, it is unknown whether it was an autoimmune reaction or simple, laser-induced, vitiligo lesions. Vitiligo Koebnerization has already been reported with Nd:YAG LHR [4], and other kinds of laser [5-7] and nonlaser mechanical hair removal [8]. No case reports of vitiligo induced by 755-nm LHR were published. In contrary, two 755-nm LHR-case reports were reassuring $[2,3]$.

Our case, with 2 episodes of 755-nm LHR-induced vitiligo lesions, is the first to report a 755-nm LHR-induced vitiligo. We should aware our vitiligo patients about the existing risk, 


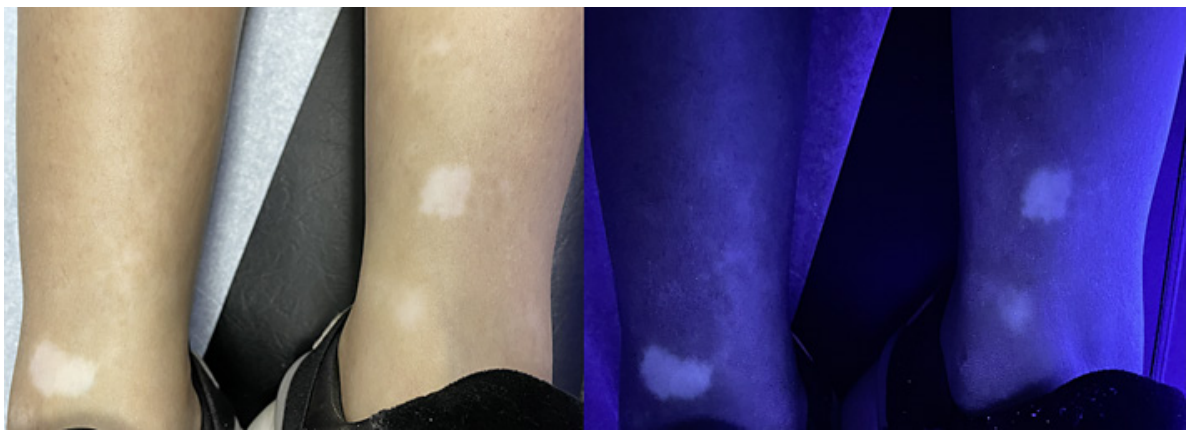

Fig. 2. Laser-induced vitiligo patches on both legs (clinical and wood's lamp) with peripheral repigmentation starting at 3 months of NB-UVB and tacrolimus $0.1 \%$ ointment.

in addition to the fact that LHR destroys the reservoir of melanocytes and stem cells, making vitiligo treatment even more difficult.

\section{Acknowledgements}

I would like to thank the deanship of scientific research, Qassim University, for funding publication of this manuscript.

\section{Statement of Ethics}

The patient has given written informed consent to publish her case (including publication of images). There is no identifiable information or pictures.

This study was reviewed and approved by the Committee of Research Ethics at Qassim University, approval number (21-02-10).

\section{Conflict of Interest Statement}

The author has no conflicts of interest to declare.

\section{Funding Sources}

There is a conditional fund by my university, Qassim University, to reimburse publication fees, if the case is accepted for publication. This fund is available for all Qassim University researchers without any influence on the work or the manuscript.

\section{Author Contributions}

Dr. Azzam Alkhalifah is the only author of this case report. He is the doctor who examined the patient and took the pictures. All parts of the manuscript were written by Dr. Azzam Alkhalifah. 


\section{Data Availability Statement}

Dr. Azzam Alkhalifah confirms that the data that support the findings of this study are available from the corresponding author (Alkhalifah.azzam@gmail.com) upon reasonable request.

\section{References}

1 Ibrahimi OA, Avram MM, Hanke CW, Kilmer SL, Anderson RR. Laser hair removal. Dermatol Ther. 2011 Feb; 24(1):94-107.

2 Zainab J. Laser hair removal in a patient with polycystic ovarian syndrome and vitiligo. J Cosmet Dermatol. 2011 Mar; 10(1):72-3.

3 Nouri K, Jimenez G, Saghari S, Keri J. Laser hair removal in a patient with vitiligo. Int J Cosmet Surg Aesthetic Dermatol. 2002 Mar 1;4(1):57-8.

4 Mavilia L, Mercuri SR. Koebner's phenomenon induced vitiligo following Nd:YAG laser epilation treatment in a woman with a past history of a Sutton nevus. Dermatol Ther. 2014 Dec;27(6):355-6.

5 Mazzotta F, Bonifazi E, Greco I. Koebner phenomenon in vitiligo after treatment with dye laser. Eur J Pediatr Dermatol. 2006 Sep 30;16(3):169.

6 Sommer S, Sheehan-Dare RA. The Koebner phenomenon in vitiligo following treatment of a port-wine stain naevus by pulsed dye laser. Br J Dermatol. 1998 Jan;138(1):200-1.

7 Kanokrungsee S, Chanprapaph K, Chaiyabutr C, Vachiramon V. A comparative study of combined treatment with fractional carbon dioxide and targeted ultraviolet B phototherapy for facial vitiligo. Lasers Med Sci. 2016 Sep;31(7):1343-9.

8 Verma SB. Vitiligo koebnerized by eyebrow plucking by threading. J Cosmet Dermatol. 2002 Dec;1(4):214-5. 